\title{
Grußwort von Dr. Ernst Breit, Vorsitzender des Historischen Vereins für Württem- bergisch Franken
}

Sehr geehrte Gäste, liebe Geschichtsfreunde,

ganz herzlich begrüße ich Sie im Namen des Historischen Vereins für Württembergisch Franken zu unserer Tagung „Aspekte der Reformation - Johannes Brenz, Primus Truber und der deutsche Südwesten“, die, veranstaltet vom Historischen Verein in Kooperation mit dem Hällisch-Fränkischen Museum, der Kunsthalle Würth und dem evangelischen Dekanat, heute und morgen in Schwäbisch Hall stattfindet.

Mein besonderer Gruß gilt unseren slowenischen Gästen, und ich freue mich, heute Abend die Botschafterin Sloweniens, Frau Marta Kos Marko, den Berater des slowenischen Staatspräsidenten für Wissenschaft und Hochschulwesen, Herrn Dr. Boštian Žekš, sowie den Referenten des heutigen Abends, Herrn Prof. Dr. Vincenc Rajšp, emeritierter Direktor des Slowenischen Wissenschaftsinstituts in Wien, in Schwäbisch Hall willkommen zu heißen.

Es ist nicht nur ihre Teilnahme an unserer Tagung, die uns zeigt, welch hohe Wertschätzung Primus Truber auch noch 500 Jahre nach seinem Wirken in ihrer Republik genießt; sie haben auch etwas mitgebracht.

Die Gedenktafel zur Erinnerung an Primus Truber und an die Druckerei von Peter Frentz, die wir dankenswerterweise heute Nachmittag am Haus von Herrn Rainer Köhnlein in der Zollhüttengasse anbringen durften, ist ein Geschenk der slowenischen Akademie der Wissenschaft. Den Verantwortlichen, insbesondere Herrn Dr. Rajšp, danken wir herzlich.

Einen Großteil unseres Wissens über die Beziehung von Truber zu Schwäbisch Hall und zu Brenz verdanken wir der fast kriminalistischen Fleißarbeit des Gothaer Buchwissenschaftlers Dr. Helmut Claus (veröffentlicht im Gutenberg-Jahrbuch 2013) und den Forschungen des leider zu früh verstorbenen Pfarrers an der Haller St. Michaelskirche und Brenz-Experten Dr. Christoph Weismann.

Dipl.-Archivarin (FH) Herta Beutter vom Hällisch-Fränkischen Museum verdankt die Truber-Forschung den Hinweis, dass mit dem fingierten Druckort „Gedruckt in Siebenbürgen“ Schwäbisch Hall gemeint ist, denn nach der Gründungssage, die um die Wende vom Mittelalter zur Neuzeit in den damals 
entstandenen Haller Chroniken von Johann Herolt und Georg Widman schriftliche Verbreitung gefunden hat, soll die Stadt aus sieben Burgen entstanden sein, die um die Solequelle errichtet worden waren. Vielleicht werden wir im Verlauf unserer Tagung noch mehr dazu erfahren.

Um ein solches Symposion durchführen zu können, braucht man nicht nur ideelle, sondern auch materielle Unterstützung, und ich danke der Stadt Schwäbisch Hall, heute hier vertreten durch Herrn Oberbürgermeister Hermann-Josef Pelgrim, und Frau C. Sylvia Weber, Direktorin der Kunsthallen Würth, deren Gäste wir heute Abend sind, für ihre großzügige Unterstützung.

Mein Dank geht auch an die Mitglieder der sogenannten Montagsrunde des Historischen Vereins für Württembergisch Franken, die bei der Konzeption, der Planung und deren Umsetzung für die Tagung aktiv beteiligt waren und sind, sowie auch an die Mitarbeiterinnen und Mitarbeiter des Hällisch-Fränkischen Museums für die gute Zusammenarbeit.

Heute und morgen beschäftigen wir uns mit der Reformation, mit Vorgängen, die sich vor 500 Jahren ereigneten.

Was können wir in dieser Tagung für das Gegenwartsverständnis in dem aufregenden Jahr 2017 - erwähnt seien hier nur die Stichworte Brexit, Donald Trump, Wahlen in den Niederlanden, in Frankreich, in der Türkei, vielleicht auch in Italien und sicher in der Bundesrepublik Deutschland - leisten?

Bei der Beantwortung dieser Frage orientiere ich mich an den Ausführungen von Jürgen Habermas über den Historiker Fritz Stern, die am 8. Februar dieses Jahres in der Frankfurter Allgemeinen Zeitung veröffentlicht worden sind. Die Antwort beginnt mit dem Hinweis auf eine Besonderheit der Beschäftigung mit Geschichte. Geschichtsdarstellungen sind immer zweifach von einem Zeitgeist geprägt:

- von der vergangenen Zeit, mit der sich Historikerinnen und Historiker befassen, - und zugleich von der Gegenwart, in der Historikerinnen und Historiker leben.

Die Referenten unserer Tagung und noch mehr wir Zuhörerinnen und Zuhörer können sich den geschichtlichen Ereignissen und Persönlichkeiten der Reformation nur aus dem Horizont des eigenen Zeit- und Weltverständnisses annähern. Die Gegenwart fließt immer in unsere Untersuchung und in unsere Wertung mit ein.

Darin liegt eine große Chance. Die Beschäftigung mit der Vergangenheit gibt uns einen Ausgangspunkt für das Verständnis der eigenen Gegenwart.

Mit dem Blick aus der Vergangenheit wird man auf Besonderheiten in der Gegenwart aufmerksam, die einem sonst nicht auffallen. Umbrüche in der eigenen Zeit, 
die einen ängstigen, lernt man unaufgeregt einzuordnen und angemessen einzuschätzen. Der Historiker erhält mit der Vergangenheit einen Spiegel, den er sich selbst und den geschichtlich interessierten Zuhörern und Lesern vorhalten kann. Das Eintauchen in eine längst vergangene Zeit hilft den Menschen heute, sich der Fundamente, auf denen sie stehen, zu vergewissern. Sie werden ihnen durch die Beschäftigung mit der Vergangenheit wieder bewusst.

Wer sich mit dem Geschehen aus früheren Jahrhunderten gründlich auseinandersetzt und sich so in die Gedanken- und Wertewelt der Vorfahren hineindenkt und hineinfühlt, erhält eine recht verlässliche Orientierung für das Zurechtfinden und das eigene Urteilen und Handeln in der Gegenwart.

Diese Behauptung lässt sich mit dem Thema unserer Tagung und sicherlich auch mit den Vorträgen heute und morgen belegen. Der Vergleich der Reformationsepoche mit unserer Zeit macht auf gesellschaftliche Spannungen aufmerksam.

Vor 500 Jahren konnte sich niemand diesen Auseinandersetzungen entziehen. Sie nahmen für viele lebensbedrohliche Ausmaße an. Der Vergleich mit der heutigen Zeit zeigt uns, dass die Lebensbedingungen damals ungleich schwieriger waren als die Konflikte, die unsere heutige Gesellschaft belasten.

Bei der Beschäftigung mit Martin Luther, Johannes Brenz, Primus Truber und deren gesellschaftlichem Umfeld relativieren sich manche Sorgen und Klagen der Gegenwart. Andererseits wachsen die Achtung und der Respekt vor den Menschen in der Reformationszeit.

Wir erleben gegenwärtig die Europäische Union in einer tiefen Krise; ihr Bestand erscheint gefährdet.

$\mathrm{Zu}$ den Ursachen für diesen Niedergang gehört auch die zumeist unzureichend ausgeprägte oder gar nicht vorhandene europäische Gesinnung vieler Bürgerinnen und Bürger der Europäischen Union. Gegenwärtig breitet sich in vielen Staaten eine nationalistische Gesinnung aus, die eine Stärkung des eigenen Nationalstaates und eine Schwächung der Europäischen Union herbeisehnt. Was lässt sich diesen Tendenzen entgegensetzen? Unsere Tagung wird es uns lehren.

Im Spiegel der geschichtlichen Ereignisse vor 500 Jahren lässt sich eine Antwort finden. Die Reformation drängte von Wittenberg (Martin Luther) und auch von Schwäbisch Hall (Johannes Brenz) aus hinaus in den europäischen Raum. Der slowenische Reformator Primus Truber ließ hier in unserer Stadt seinen Katechismus und das Abecedarium in der Landessprache drucken.

Dieser Vorgang zeigt: Die Reformation 1517 war nicht in erster Linie geprägt von ökonomischen Kosten-Nutzen-Relationen, die heute unser Denken mehr be- 
stimmen, als wir uns dessen bewusst sind. 1517 ging es bei der Reformation um Höheres, und auch in der Europäischen Union geht es eigentlich um mehr als um Gewinnsteigerung.

Die Auseinandersetzung mit der Geschichte bei unserer Tagung führt uns die Reformation als eine Wertegemeinschaft vor Augen, die - wie das Beispiel Brenz-Truber zeigt - in den europäischen Raum hineingewirkt hat.

Auch wenn sich in diesen 500 Jahren die Werte verändert haben, ich denke z. B. an Säkularisation und Demokratie, Menschenwürde und Menschenrechte, so hält uns die Reformation mit Brenz und Truber einen Spiegel vor, in dem wir uns selbst mit unseren Versäumnissen sehen können.

Das Bewusstsein einer Wertegemeinschaft aber hat zwischen den europäischen Regierungen ebenso wie unter den Bürgerinnen und Bürgern an Kraft verloren.

Die Arbeit an der Geschichte der Reformation und ihrer Persönlichkeiten kann diese Ideen für die Gegenwart neu beleben.

Wenn dies geschieht, dann beweist sich wieder die alte Weisheit:

„Die Zukunft hat eine lange Vergangenheit!“ 